\title{
MANEJO QUIRÚRGICO Y COMPLICACIONES DE LA I.U.E: NUESTRA EXPERIENCIA EN 385 PACIENTES OPERADAS EN LOS ÚLTIMOS 25 AÑOS
}

\author{
D. GARCÍA FLÓREZ, P. PÉREZ SANZ, G. BRIONES MARDONES, B. POZO MENGUAL, \\ R. GARCÍA NAVAS, L. PERALES CABANAS*, E. FERNÁNDEZ FERNÁNDEZ*, \\ A. ESCUDERO BARRILERO**
}

Servicio de Urología. *Sección de Urodinámica. Hospital Ramón y Cajal. Madrid.

Actas Urol Esp. 27 (2): 92-96, 2003

\section{RESUMEN \\ "MANEJO QUIRÚRGICO Y COMPLICACIONES DE LA I.U.E.: NUESTRA EXPERIENCIA EN 385 PACIENTES OPERADAS EN LOS ÚLTIMOS 25 AÑOS"}

Se presentan los resultados y complicaciones obtenidos y comparados en dos grandes series de pacientes operadas de Incontinencia Urinaria de Esfuerzo (I.U.E) en nuestro servicio. Desde enero de 1994 a diciembre de 2001 hemos recogido 195 pacientes con una edad media de 57,9 y las hemos comparado con una serie previa de 189 pacientes que comprende desde enero de 1976 hasta diciembre de 1993 con una edad media de 53,6 años. La tasa de pacientes continentes en ambas series fue similar $(70,8 \%$ pre- 94 y $74,5 \%$ post-94), leve incontinente en $22,7 \%$ pre-94 y $19,2 \%$ post-94 e incontinente $6,3 \%$ pre-94 y $6,2 \%$ post-94.

La técnica de Burch es la más empleada y corresponde a la más eficaz en el estudio con 113 pacientes y un $74,3 \%$ de pacientes continentes en la primera serie y 144 pacientes con $77 \%$ continentes en la segunda serie.

Las complicaciones más frecuentes fueron las mismas en las dos series: infección del tracto urinario ( $5,2 \%$ y $9 \%$ en primera y segunda serie) e infección de herida quirúrgica ( $4,2 \%$ y $2,2 \%$ respectivamente).

Concluimos que a pesar de la introducción de nuevas y útiles técnicas correctoras (TVT, inyección de sustancias periuretrales...) la mayor tasa de éxito a largo plazo continúa siendo la colposuspensión tipo Burch.

PALABRAS CLAVE: Incontinencia urinaria de esfuerzo. Burch. Técnica quirúrgica.

\section{ABSTRACT \\ "SURGICAL MANAGEMENT AND URINARY STRESS INCONTINENCE COMPLICATIONS: OUR EXPERIENCE IN 385 FEMALE OPERATED PATIENTS DURING THE LAST 25 YEARS"}

We present the results and complications found and compared in 2 large series of patients who underwent a surgical procedure to avoid Urinary Stress Incontinence in our hospital. From january of 1994 to december of 2001 we collected 195 patients with an average age of 57.9 y we compared them with a prior series of 189 patients collected between january of 1976 and december of 1993,with an average age of 53.6. The continence rate was similar in both series $(70.8 \%$ pre-94 and $74.5 \%$ post 94$)$, slight incontinence $22.7 \%$ pre-94 and $19.2 \%$ post-94 and for complete incontinence $6.3 \%$ pre- 94 and $6.2 \%$ post- 94 .

The Burch procedure was the most common and also shows the greatest effectivness in our trial involving 113 cases and a continence rate of $74.3 \%$ in the first series and 144 cases and $77 \%$ respectively in the second series.

The most common complications were very similar in both groups: tract urinary infection $(5.2 \%$ and $9 \%$ first and second group), surgical wound infection ( $4.2 \%$ and $2.2 \%$ respectively).

We conclude that in spite of the introduction of new and useful surgical procedure (TVT, periurethal substances injection...) the greatest long term succes rate still corresponds to the Burch colposuspension. KEY WORDS: Urinary stress incontinence. Burch. Surgical procedure. 
$\mathrm{L}$ a incontinencia de orina aunque se considera un sintoma más que una enfermedad representa un importante impacto emocional y social sobre el individuo, sus familiares y las personas que le rodean; de este modo induce a la pérdida de autoestima y a mermar la capacidad de un estilo de vida independiente.

Se puede decir que la Incontinencia Urinaria en términos generales afecta a unos dos millones de personas en España y a casi 13 millones en los EE.UU. El pico de incidencia se sitúa en la tercera década. La tasa de prevalencia varía según la población de estudio, la definición de incontinencia y la forma de obtener la información; podemos decir que en $>60$ a en varones oscila entre 5-15\% y en mujeres varía entre $10-30 \%$. En personas autónomas >60a la prevalencia es del 15-35\% con una proporción mujeres/varones 2:1 y finalmente en personas mayores encamadas aumenta hasta el 53\% de la población.

\section{MATERIAL Y MÉTODOS}

Desde enero de 1976 a diciembre de 1993 se han recogido 189 pacientes operadas de I.U.E con una edad media de 53,6 años (rango 17-79a) y un tiempo medio de seguimiento de 22 meses (rango 6-120 meses). Un 67,7\% de los pacientes (128 casos) tenían cierto grado de cistocele.

En nuestra segunda serie hemos recogido, desde enero de 1994 hasta diciembre de 2001, 195 pacientes con una edad media de 57,9 años (rango entre 28-79 años) y un tiempo medio de seguimiento de 19,7 meses (rango 3-132 meses). Un $74 \%$ de las pacientes (131 casos) presentaban un grado de cistocele previo a la cirugía.

La técnica quirúrgica más empleada en la primera serie (1976-1993) fue la colposuspensión tipo Burch con 113 casos $(56,7 \%)$, después la de Marshall-Marchetti-Krantz (MMK) con 24 casos (12\%), la de Raz con 23 casos (11,5\%), técnica de Kelly con 20 casos (10\%), técnica de Stamey con 10 casos (5\%) y técnicas de Sling con 9 casos $(4,5 \%)$. Hubo 8 pacientes con múltiples cirugías de incontinencia pero que no fueron incluidas en esta serie.

En la segunda serie (1994-2001) la técnica más empleada vuelve a ser la tipo Burch en 144 casos $(81,3 \%)$, seguida de la TVT con 20 casos $(11,2 \%)$, Raz en 11 casos $(6,2 \%), 1$ caso de MMK y otro tipo
Kelly. Se contabilizaron 14 implantes de prótesis de incontinencia y 4 pacientes con varias cirugías de incontinencia pero tampoco fueron analizados en este estudio.

\section{RESULTADOS}

En términos globales hemos obtenido los siguientes resultados en cuanto a continencia integral comparando los dos grupos de pacientes (Tabla I):

En la primera serie:

Continencia completa en 134 casos (70,8\%), leve incontinencia en 43 casos $(22,7 \%)$ e incontinencia en 12 casos (6,3\%). El análisis pormenorizado según técnica quirúrgica podemos verlo reflejado en la Tabla II.

\section{TABLA}

\begin{tabular}{|l|c|c|c|}
\hline & Continente & Leve incont. & Incontinente \\
\hline $\begin{array}{l}1^{\text {a }} \text { serie } \\
(1976-1993)\end{array}$ & $70,8 \%$ & $22,7 \%$ & $6,3 \%$ \\
\hline $\begin{array}{l}2^{\text {a }} \text { serie } \\
(1994-2001)\end{array}$ & $74,5 \%$ & $19,2 \%$ & $6,2 \%$ \\
\hline
\end{tabular}

TABLA II

\begin{tabular}{|l|c|c|c|}
\hline & Continente & Leve incont. & Incontinente \\
\hline $\begin{array}{l}\text { Burch } \\
(113 \text { casos })\end{array}$ & $84(74,3 \%)$ & $26(23 \%)$ & $3(2,6 \%)$ \\
\hline $\begin{array}{l}\text { MMK } \\
(24 \text { casos })\end{array}$ & $18(75 \%)$ & $3(12,5 \%)$ & $3(12,5 \%)$ \\
\hline $\begin{array}{l}\text { Raz } \\
(23 \text { casos })\end{array}$ & $17(73,9 \%)$ & $4(17,3 \%)$ & $2(8,6 \%)$ \\
\hline $\begin{array}{l}\text { Kelly } \\
(20 \text { casos })\end{array}$ & $9(45 \%)$ & $9(45 \%)$ & $2(10 \%)$ \\
\hline $\begin{array}{l}\text { Stamey } \\
(10 \text { casos })\end{array}$ & $7(70 \%)$ & $1(10 \%)$ & $2(20 \%)$ \\
\hline $\begin{array}{l}\text { Sling } \\
(9 \text { casos })\end{array}$ & $6(66,6 \%)$ & $1(11,1 \%)$ & $2(22,2 \%)$ \\
\hline
\end{tabular}

Residuos altos (>100 cc) tras la cirugía en 51 casos (26,9\%): Burch 25 casos, Raz 12 casos, Sling 6 casos, Stamey 2 casos, Kelly 3 casos y MMK 3 casos.

Retención completa tras la cirugía en 14 casos (7,4\%): Raz 7 casos, Burch 4 y Sling 3 casos y ninguno en técnicas de Kelly, MMK y Stamey. 
Retención completa y presión substraida miccional:

$<15$ cc de $\mathrm{H} 20$ en 10 casos.

$>15$ cc de $\mathrm{H} 20$ en 4 casos.

Residuo alto y presión substraída miccional:

$<15$ cc de $\mathrm{H} 20$ en 34 casos.

$>15$ cc de $\mathrm{H} 20$ en 17 casos.

En esta serie encontramos 77 pacientes con distintos grados de urgencia precirugía y 34 casos con urgencia post-quirúrgica que corresponden a:

- Obstrucción 8 casos.

- Urgencia sensorial 21 casos.

- Inestabilidad 5 casos.

Se encontraron 16 pacientes $(8,4 \%)$ sin urgencia pre y con urgencia post-cirugía.

En nuestra segunda serie (1994-2001) hemos obtenido:

Continencia completa en 132 casos $(74,5 \%)$, leve incontinencia en 34 casos $(19,2 \%)$ e incontinencia en 11 casos (6,2\%). El análisis esquemático es (Tabla III):

\section{TABLA III}

\begin{tabular}{|l|c|c|c|}
\hline & Continente & Leve incont. & Incontinente \\
\hline $\begin{array}{l}\text { Burch } \\
(144 \text { casos })\end{array}$ & $111(77 \%)$ & $25(17,3 \%)$ & $8(5,5 \%)$ \\
\hline $\begin{array}{l}\text { TVT } \\
(20 \text { casos })\end{array}$ & $13(65 \%)$ & $6(30 \%)$ & $1(5 \%)$ \\
\hline $\begin{array}{l}\text { Raz } \\
(11 \text { casos })\end{array}$ & $8(72,7 \%)$ & $3(27,7 \%)$ & $0(0 \%)$ \\
\hline $\begin{array}{l}\text { MMK } \\
(1 \text { caso })\end{array}$ & $0(0 \%)$ & $1(100 \%)$ & $0(0 \%)$ \\
\hline $\begin{array}{l}\text { Kelly } \\
(1 \text { caso })\end{array}$ & $0(0 \%)$ & $0(0 \%)$ & $1(100 \%)$ \\
\hline
\end{tabular}

Residuos altos tras la cirugía en 33 casos (18,6\%): Burch 29, Raz 4 y ningún caso en las técnicas de Kelly, MMK y TVT.

Retención completa tras la cirugía en 12 casos (6,7\%): Burch 8, Raz 3, TVT 1 y ningún caso en Kelly, MMK y Stamey.

Retención completa y presión substraída miccional:

$<15$ cc de $\mathrm{H} 20$ en 8 casos.

$>15$ cc de $\mathrm{H} 20$ en 5 casos.
Residuo y presión substraída miccional:

$<15$ cc de $\mathrm{H} 20$ en 20 casos.

$>15$ cc de $\mathrm{H} 20$ en 14 casos.

En la segunda serie encontramos 55 pacientes con distintos grados de urgencia precirugía y 42 casos con urgencia post-quirúrgica de las cuales:

- Obstrucción en 8 casos.

- Urgencia sensorial en 32 casos.

- Inestabilidad en 5 casos.

Hubo 22 casos sin urgencia previa a la intervención que luego desarrollaron urgencia postquirúrgica.

El resultado del análisis de las complicaciones se puede ver en la Tabla IV.

\section{TABLA IV}

\begin{tabular}{||l|c|c|}
\hline & 1a Serie & 2a Serie \\
\hline I.T.U. & $10(5,2 \%)$ & $16(9 \%)$ \\
\hline Infección de herida $\mathrm{Q}(\mathrm{x})$ & $8(4,2 \%)$ & $4(2,2 \%)$ \\
\hline Obstrucción ureteral & $8(4,2 \%)$ & $3(1,6 \%)$ \\
\hline Eventración & $3(1,5 \%)$ & $3(1,6 \%)$ \\
\hline TVP y/o TEP & $3(1,5 \%)$ & $1(0,5 \%)$ \\
\hline
\end{tabular}

\section{DISCUSIÓN}

La incontinencia urinaria de esfuerzo afecta como hemos visto a un sector importante de la población y en su mayoría del sexo femenino. Existen varios factores implicados en su etiopatogenia como son los factores anatómicos, neurológicos así como los antecedentes de la paciente (cirugía previa del ap.g-u, obesidad, edad, paridad...etc).

El manejo de la I.U.E femenina es fundamentalmente quirúrgico existiendo diversas técnicas para su corrección. Cuando se plantean tantas técnicas quirúrgicas para solucionar un problema es lógico pensar que ninguna de ellas es buena en términos absolutos; pero, si somos capaces de apurar al máximo en el diagnóstico, podremos realizar un tratamiento a medida, seleccionando aquella técnica que pueda aportar mayor ventaja a la paciente ${ }^{1}$. En nuestro servicio hemos empleado las técnicas anteriormente mencionadas (Burch, Raz, MMK...) con diferentes tasas de éxito, así como, otras más novedosas (TVT) cuyos resultados compararemos con otros estudios. 
La elección de una técnica u otra dependerá de las características personales de cada caso. Tenemos en cuenta la edad, cirugías previas (sobre todo del suelo pélvico), historia obstétrica, pasando por el diario y clínica miccional, exploración física (incluyendo examen neurológico) para después realizar las pruebas urodinámicas pertinentes. Los parámetros más útiles son los obtenidos durante la cistomanometría, el test de presión-flujo y los perfiles de presión uretral ${ }^{2}$.

Es importante con todo ello determinar si existe hipermotilidad uretral, déficit esfinteriano intrínseco y/o inestabilidad del detrusor porque esto nos orientará a elegir una técnica quirúrgica $\mathrm{u}$ otra ${ }^{3}$. Unificando la terminología podemos agruparlas de inicio en cuatro grandes grupos ${ }^{4}$ que serán: la suspensión retropúbica, la suspensión transvaginal, la reparación anterior y los procedimientos de sling o cabestrillo. Dicho esto nos centraremos en comentar nuestros resultados comparándolos con los de la literatura.

Los datos más favorables que hemos obtenido han sido en la colposuspensión tipo Burch y en la técnica del TVT, coincidiendo estas técnicas de suspensión retropúbica; de cabestrillo como las más exitosas en los artículos que hemos revisa$\mathrm{do}^{2,5-10}$. Algo menos efectiva fue la suspensión transvaginal aunque en nuestra serie casi se igualó con la retropúbica y ya por debajo se situaron otras técnicas de reparación anterior, en relación con la corrección de prolapsos vaginales. La inyección de sustancias periuretrales (teflón, colágeno..) en nuestra experiencia hemos obtenido resultados muy variables. Estos no se han analizado debido a esta disparidad y al pequeño número de casos que hemos encontrado.

Múltiples factores pueden analizarse y ser considerados indicadores pronósticos tanto en las complicaciones a corto y medio plazo (retención completa de orina, residuos altos, pérdidas de sangre, infecciones..) como en los resultados favorables en cuanto a continencia global a largo plazo.

En nuestro artículo relacionamos el valor urodinámico de la $\mathrm{P}$. substraída miccional $<15 \mathrm{cc}$ de agua con unos residuos post-cirugía más altos y con un mayor índice de episodios de retención completa de orina. Otros autores mencionan como factores pronósticos adversos: la incontinencia mixta $^{11}$, la edad (>69 años), la cirugía de incontinencia previa, morbilidad post-operatoria febril, las dificultades miccionales post-operatorias inmediatas $^{12}$ y la presión uretral mínima de fuga $<25 \mathrm{cc}$ de agua ${ }^{3,13}$. También son recogidos como influyentes el sobrepeso (>80 kg) y la pérdida de sangre perioperatoria $>1.000 \mathrm{cc}$ entre otros ${ }^{14}$.

Sobre las complicaciones creemos que no existen datos fiables en los diferentes estudios sugiriendo en los prospectivos que no existen grandes diferencias entre las distintas técnicas correctoras. Las complicaciones más frecuentes recogidas son la infección de orina e infección de herida quirúrgica, dificultades miccionales e inestabilidad del detrusor $^{6}$. A pesar de lo comentado podemos señalar que en general encontramos una mayor tasa de complicaciones en la suspensión retropúbica y en los procedimientos de Sling ${ }^{5}$. Estas dos técnicas suponen un tiempo de estancia hospitalaria mayor que hoy en día podría verse reducida con el uso de técnicas laparoscópicas ${ }^{14}$, aunque esto incrementaría el coste global de la intervención quirúrgica y no está exenta de complicaciones ${ }^{15}$ : intraoperatorias (perforación vesical, desgarro peritoneal, enfisema subcut...) y post-operatorias (hematoma del lecho y/o pared e infección de pared).

\section{CONCLUSIONES}

Desde el punto de vista urodinámico llegamos a la conclusión en nuestro estudio que los pacientes con una P. substraída miccional del detrusor $<15 \mathrm{~cm}$ de agua eran más propensos a desarrollar residuos altos y/o retención aguda de orina tras la cirugía que los que tenían una P. detrusor $>15$ cc de agua.

Las complicaciones más frecuentes en nuestras series como en las de la literatura vienen a ser la infección de aparato urinario (5,2-9\%), en relación con la manipulación de la vía urinaria y en menor medida la infección de herida quirúrgica (2,2-4,2\%), cuando el abordaje de la técnica era transcutáneo-mucoso.

Una vez analizadas y comparadas las dos series de pacientes intervenidas de I.U.E (Pre-94 y Post 94) se obtuvieron resultados superponibles, con la salvedad que técnicas correctoras como la de Raz o la de Kelly apenas se emplean en favor de otras como la de TVT o la inyección de sustancias periuretrales, aunque a pesar de estas últimas la colposuspensión tipo Burch es la que representa un mayor índice de resultados favorables a largo plazo en cuanto a continencia post-quirúrgica. 


\section{REFERENCIAS}

1. POSTIUS ROBERT J, CASTRO DÍAZ D, BAÑARES BAUDET F.: Incontinencia urinaria de esfuerzo femenina. Urología 1998; 4 (3).

2. TALLADA M, ESPEJO E, CÓZAR JM, VICENTE FJ.: Investigación urodinámica. En: Incontinencia urinaria.Vigo: Fundación de estudios y formación sanitaria, 2001.

3. DROUIN J, TESSIER J, BERTRAND PE, SCHICK E.: Burch colposuspensión: long-term results and review of published reports. Urology 1999; 54: 808-814.

4. LEACH GE, DMOCHOWSKI RR, APPELL RA et al.: Female stress urinary incontinence clinical guidelines panel summary report on surgical management of female stress urinary incontinence. The Journal of Urology 1997 sept; 158: 875-880.

5. BLACK NA, DOWNS SH.: The effectiveness of surgery for stress incontinence in women: a sistematic review. British Journal of Urology 1996; 78: 497-510.

6. PARK GS, MILLER EJ.: Surgical treatment of stress urinary incontinence: a comparison of the Kelly plication, MMK and Pereyra procedures. Obstetric Gynecol 1988; 71: 575-579.

7. KLUTKE JJ, KLUTKE CG, BERGMAN J et al.: Urodinamic changes in voiding after antiincontinence surgery: an insight into the mechanism of cure. Urology 1999 june; 54 (6): 1.003-1.007.

8. BERGMAN A, KOONINGS PP, BELLARD CA.: Primary stress urinary incontinence and pelvic relaxation: prospective randomized comparison of three diferent operations. Am J Obstet Gynecol 1989; 161: 97-101.

9. DEBORATH J. LIGHTNER, MD et cols.: Treatment options for women with stress urinary incontinence. Mayo Clin Proc 1999; 74: 1.149-1.156.
10. ERIKSEN BC, HAGAN B, EIK-NES ST et al.: Longterm effectiveness of the Burch colposuspension in female urinary stress incontinence. Acta Obstetric Gynecol Scand 1990; 69: 45-50.

11. JOLHEDA KP, RIDEN G.: Prosnostic factors and long-term results of the Burch colposuspension: a retrospective study. Acta Obstet Gynecol Scand 1994; 73: 642-647.

12. KINN A-C.: Burch colposuspension for stress urinary incontinence: 5 years results in 153 women. Scand J Urol Nephol 1995; 29: 449-455.

13. ALCALAY M, MARGA A, STANTON SL.: Burch colposuspension: a 10-20 years follow up. $\mathrm{Br} J$ Obstet Gynecol 1995; 102: 740-745.

14. KOHLI N MD, JACOBS PA MD, EDDIE, SZE HM MD, ROAT TW, KARRAM MM MD.: Open compared with laparoscopic approach to Burch colposuspension: a cost analysis. Obstetrics and Gynecology 1997 sept; 90 (3): 411-415.

15. DE PALACIO ESPAÑA A, HERNÁNDEZ FERNÁNDEZ C.: Colposuspensión laparoscópica. Servicio de Urología del Hospital Gregorio Marañón. Madrid. Atlas de Incontinencia Urinaria: 277-284.

\author{
Dr. D. García Flórez \\ C/ Generosidad, 14 - portal C, 1ㅇ A \\ 28014 Madrid
}

(Trabajo recibido el 10 abril 2002) 